\title{
The effect of chlorpromazine on the depression effect
}

\begin{abstract}
The hypothesis that the depression effect is produced by increased emotionality resulting from a downward shift in reward was tested by studying the effects of a downward shift in reward in control animals and animals under the influence of chlorpromazine. Differences between control and drug groups appeared only in the preshift data, the depression effect appearing in both control and drug groups.
\end{abstract}

\section{Problem}

One of the most reliable phenomena in ratpsychology is the depression effect. If two groups of animals are trained to run down a runway to food reward, one to a large reward and the other to a small reward, the large reward group comes to run at a higher asymptotic speed than the small reward group. If the large reward group is then shifted to the same amount of reward as the small reward group, the speed of the animals declines over trials until it falls significantly below that of the unshifted small reward animals. Some investigators have suggested that this phenomenon is emotionally based. Crespi (1944) held that encountering a reward smaller than that expected produced an emotional reaction of anger which reduced the vigor of the goal directed response. Seward (1951) proposed a similar hypothesis in the context of an S-R theory: a downward shift in amount of reward leads to a blocking of the $R_{g}$ originally conditioned to the goal-box stimuli, producing frustration which acts as a negative drive and subtracts from the positive drive to food. If the depression effect is, in fact, the product of increased emotionality, then an induced reduction of emotionality should lead to attenuation or elimination of the depression effect.

In this experiment, an initial test of this hypothesis is carried out by comparing the effects of a downward shift in reward in control animals and animals under the effects of chlorpromazine. Studies of drug effects in traumatic learning situations suggest that chlorpromazine acts to alleviate emotionality. In an experiment which controlled motor depression effects, Gonzales \& Shepp (1962) found that chlorpromazine inhibited the learning of an avoidance response. In a discrimination experiment with punishment for errors, Gonzales \& Ross (1961) found that animals under the effects of chlorpromazine adjusted to successive habitreversals more rapidly than control animals.

Method

The apparatus consisted of a starting-box ( 5 by 9 by 6 in), a runway ( 5 by 93 by 6 in), all made of wood and painted grey with hinged Plexiglas tops. The goal-box was placed at a right angle to the end of the runway and was entered by a right turn. Two guillotine doors separated the starting-box and goal-box from the runway. When the starting-box door was raised, a microswitch, mounted above the door, was operated starting a Standard Electric Timer. When a beam of light passing across the runway 84 in from the starting-box door was broken, the timer stopped. Times were recorded in .01 sec. and transformed to logs.

The Ss were 24 male albino rats of the Wistar strain between 88 and 116 days old at the beginning of the experiment. Their body weights were reduced to $85 \%$ of ad lib feeding weight. They were divided into four matched groups of six each on the basis of body weight. The groups were designated .2-.2 Control, 1.6 - .2 Control, .2 - .2 Drug, and 1.6 - .2 Drug. Seven days before the experiment began, the drug groups were daily fed $10 \mathrm{mg} / \mathrm{kg}$ of chlorpromazine hydrochloride mixed in wet mash. The control groups were fed wet mash with no drug in it.

Each $S$ was given one trial per day throughout the experiment. For the first 20 trials, one control and one drug group was rewarded with $.2 \mathrm{gm}$ of wet mash and the other control and drug groups with $1.6 \mathrm{gm}$ of wet mash. On trials 21-31, the groups receiving $1.6 \mathrm{gm}$ were shifted to $.2 \mathrm{gm}$ while those groups receiving .2 gm continued to be rewarded with the same amount. One-half hr. after each day's trial, Ss were fed enough wet mash and hard pellets to maintain them at their experimental weight. The drug groups were fed wet mash containing chlorpromazine while the control groups were fed regular wet mash.

\section{Resuits}

In Fig. 1, all four groups show negatively accelerated acquisition curves reaching asymptote on trials 12-20. The asymptotic difference in log time between the large and small reward control groups is relatively large compared to the difference between the large and small reward drug groups. An analysis of variance performed on the mean log times over trials 12-20 shows a significant Groups-effect $(F=6.78, d f=3,20)$. An orthogonal comparison between the .2 and 1.6 control groups revealed a significant difference $(F=14.34, \mathrm{df}=1,20)$, but a comparison between the .2 and 1.6 drug groups is not significant $(F=2.22$, $d f=1,20)$.

Following the shift in reward, the curves of the shifted control and drug groups rise gradually to the level of the unshifted animals. On Day 26, the 1.6-.2 


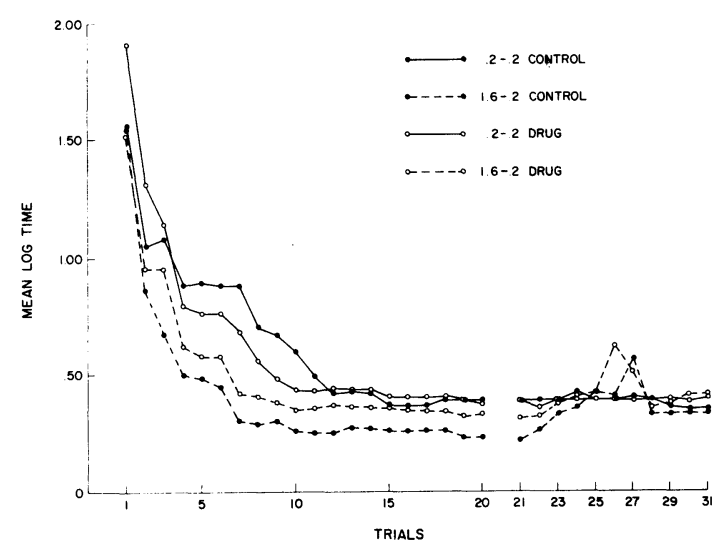

Fig. 1. Preshift- and postshift-in-reward curves for control and drug grouns.

Drug Group curve rises markedly above the curves of the unshifted groups, while on Day 27, the 1.6-.2 Control Group curve shows a similar rise. By Day 28 , the curves of both shifted groups have returned to the level of the unshifted groups and remain there for the remaining three days of the experiment. An analysis of variance on Day 26 times shows a significant Groupseffect ( $F=4.06, d f=3,20)$, and an orthogonal comparison between the 1.6-.2 Drug Group and the other three groups combined is significant $(F=12.13, d f=1,20)$. An analysis of the Day 27 scores yields an F-ratio for groups significant at about the .06 level ( $F=3.00$, $\mathrm{df}=3,20$ ), and orthogonal comparisons show that the difference between groups 1.6-.2 Control and .2-.2 Control is significant $(F=5.64, d f=1,20)$, but the difference between groups 1.6-.2 Drug and .2-.2 Drug falls short of significance $(F=2.94, \mathrm{df}=1,20)$. A final analysis of variance on the mean log times over days 28-31 yields an insignificant Groups-effect $(\mathrm{F}<1.00)$.

\section{Diseussion}

Control and drug groups differed in this experiment only on preshift acquisition trials, where amount of reward significantly differentiated control groups but not drug groups. This finding can be accounted for in terms of motor depression; that is, chlorpromazine had the effect of placing an upper limit on the speed with which drug animals could approach reward.

The depression effect appeared in both control and drug groups, and the point in time, magnitude, and duration of the effect was equivalent in both groups. There is no suggestion in these data that the factors which produce the depression effect are modified by chlorpromazine. Before the hypothesis that the depression effect is emotionally based should be rejected, however, further studies of the depression effect should be made using different drugs and varying dosage levels.

One other point which should be noted is that the depression effect in this experiment was highly transient, lasting for only one day in each of the shifted groups. Gonzalez, Gleitman, \& Bitterman (1962) also found the depression effect to disappear with continued training, but that effect was not as short-lived as this one.

\section{References}

Crespi, L. P. Amount of reinforcement and level of performance. Psychol. Rev., 1944, 51, 341-357

Gonzalez, R. C., Gleitman, H., \& Bitterman, M. E. Some observations on the depression effect. J. cump. physiol. Psychol., 1962, $55,578-581$.

Gonzalez, R. C., \& Ross, S. The effects of chlorpromazine on the course of discrimination-reversal learning in the rat. J. comp. physiol. Psychol., 1961, 54, 645-648.

Gonzalez, R. C., \& Shepp, B. The effects of chlorpromazine on instrumental learning based on conditioned fear. Canad. J. Psychol., 1962, 16, 64-71.

Seward, J. P. Experimental evidence for the motivating function of reward. Psychol. Bull., 1951, 48, 130-149. 